\title{
Adaptive output regulation of uncertain nonlinear systems with unknown control directions
}

DOI:

10.1007/s11432-018-9520-2

\section{Document Version}

Accepted author manuscript

Link to publication record in Manchester Research Explorer

\section{Citation for published version (APA):}

Chen, J., Li, Z., \& Ding, Z. (2018). Adaptive output regulation of uncertain nonlinear systems with unknown control directions. Science China. Information Sciences. https://doi.org/10.1007/s11432-018-9520-2

\section{Published in:}

Science China. Information Sciences

\section{Citing this paper}

Please note that where the full-text provided on Manchester Research Explorer is the Author Accepted Manuscript or Proof version this may differ from the final Published version. If citing, it is advised that you check and use the publisher's definitive version.

\section{General rights}

Copyright and moral rights for the publications made accessible in the Research Explorer are retained by the authors and/or other copyright owners and it is a condition of accessing publications that users recognise and abide by the legal requirements associated with these rights.

\section{Takedown policy}

If you believe that this document breaches copyright please refer to the University of Manchester's Takedown Procedures [http://man.ac.uk/04Y6Bo] or contact uml.scholarlycommunications@manchester.ac.uk providing relevant details, so we can investigate your claim.

\section{OPEN ACCESS}




\title{
Adaptive output regulation of uncertain nonlinear systems with unknown control directions
}

\author{
Jingyu CHEN, Zhenhong LI \& Zhengtao DING* \\ School of Electrical and Electronic Engineering, University of Manchester, Sackvile Street Building, Manchester M13 9PL, UK
}

Citation Chen J Y, Li Z H, Ding Z T. Adaptive output regulation of uncertain nonlinear systems with unknown control directions. Sci China Inf Sci, for review

Dear editor,

The output regulation problem for nonlinear systems with unknown exosystems has been an important research topic over the last few decades [1]. The early results commonly assume that the sign of the high-frequency gain is known. It is shown [2] that a nonlinear system can be transformed into a special observer form under geometric coordinatefree conditions. In this case, with a known control direction, the problem can be solved by a robust control method such as high-gain feedback. However, this method is not applicable when the highfrequency gain sign is unknown. It has been proved that the standard way to deal with the unknown sign of the high-frequency gain is the Nussbaum gain technique [3]. The Nussbaum gain is a kind of oscillating function which allows the controller to try both positive and negative directions. It is still a challenge to solve the nonlinear output regulation problem with both unknown nonlinear exosystems and unknown high-frequency gain sign. This motivates us to develop a new approach, which combines the circle criterion with Nussbaum gain. Recently, this problem has attracted extensive attention. In [4], the Nussbaum gain technique is integrated with the backstepping method for solving adaptive consensus output regulation of a class of network-connected nonlinear systems, and some progresses are reported on cooperative output regulation of a class of nonlinear multi-agent systems [5] without any knowledge of the high frequency gain [6].

In this letter, we consider adaptive output regulation of output feedback systems with both unknown nonlinear exosystems and unknown highfrequency gain sign. A nonlinear adaptive internal model is proposed on a basis of circle criterion to reproduce the feedforward input term. As for the nonlinear exosystems, some specific assumptions are determined such that the circle criterion can be used in the stability analysis for the proposed internal model. Because of the lack knowledge of parameters in the system and the exosystem, a Nussbaum gain is then used to tackle the unknown sign of high-frequency gain. The proposed internal model, Nussbaum gain and nonlinear adaptive backstepping technique together provide a solution to the output regulation with nonlinear exosystems. The control input is finally obtained by a recursive procedure. The proposed control scheme guarantees the global asymptotic convergence of the tracking error.

Problem statement. We consider a single-inputsingle-output nonlinear system which can be transformed into the output feedback form

$$
\begin{aligned}
\dot{\zeta} & =A_{c} \zeta+\phi(y) a+E(\omega)+b u, \\
y & =C \zeta, \\
e & =y-q(\omega),
\end{aligned}
$$

\footnotetext{
*Corresponding author (email: zhengtao.ding@manchester.ac.uk)
} 


$$
A_{c}=\left[\begin{array}{ccccc}
0 & 1 & 0 & \ldots & 0 \\
0 & 0 & 1 & \ldots & 0 \\
\vdots & \vdots & \vdots & \ddots & \vdots \\
0 & 0 & 0 & \ldots & 1 \\
0 & 0 & 0 & \ldots & 0
\end{array}\right], b=\left[\begin{array}{c}
0 \\
\vdots \\
b_{\rho} \\
\vdots \\
b_{n}
\end{array}\right], C=\left[\begin{array}{c}
1 \\
0 \\
\vdots \\
0
\end{array}\right]^{T},
$$

where $\zeta \in \mathbb{R}^{n}$ is the state vector, $u \in \mathbb{R}$ is the control input, $y \in \mathbb{R}$ is the output, $e \in \mathbb{R}$ is the measurement output, $a \in \mathbb{R}^{m}$ and $b \in \mathbb{R}^{n}$ are vectors of unknown parameters, $b_{\rho} \neq 0$ indicates that the nonlinear system has a constant relative degree of $\rho, E(\omega): \mathbb{R}^{l} \rightarrow \mathbb{R}^{n}$ is an unknown nonlinear function of $\omega, \phi: \mathbb{R} \rightarrow \mathbb{R}^{n \times m}$ is a smooth nonlinear vector field with $\phi(0)=0$ and $\|\phi(y)-\phi(\hat{y})\| \leqslant M \sigma(|y-\hat{y}|)|y|$ and $M$ is an unknown positive constant, and $\sigma(\cdot) \in \mathcal{K}$ is a known smooth function, $q$ is an unknown polynomial of $\omega$, and $\omega \in \mathbb{R}^{l}$ are disturbances, and they are generated from an unknown nonlinear exosystem

$$
\dot{\omega}=s(\omega) .
$$

Assumption 1. The system is minimum phase, i.e., the polynomial $\mathcal{B}(s)=\sum_{i=\rho}^{n} b_{i} s^{n-i}$ is Hurwitz, and the sign of the high-frequency gain $b_{\rho}$ is unknown.

Assumption 2. The flows of vector field $s(\omega)$ are bounded and converge to periodic solutions. State transformation. Define an input filer

$$
\begin{aligned}
\dot{\xi}_{i} & =-\lambda_{i} \xi_{i}+\xi_{i+1}, \quad \text { for } i=1, \ldots, \rho-2, \\
\dot{\xi}_{\rho-1} & =-\lambda_{\rho-1} \xi_{\rho-1}+u
\end{aligned}
$$

where $\lambda_{i}>0$ for $i=1, \ldots, \rho-1$ are the design parameters, and define the filtered transformation $\bar{x}=\zeta-\sum_{i=1}^{\rho-1} \bar{d}_{i} \xi_{i}$, where $\bar{d}_{i} \in \mathbb{R}^{n}$ for $i=1, \ldots, \rho-1$ and they are generated recursively by

$$
\begin{aligned}
\bar{d}_{\rho-1} & =b, \\
\bar{d}_{i} & =\left(A_{c}+\lambda_{i+1} I\right) \bar{d}_{i+1}, \quad \text { for } i=1, \ldots, \rho-2 .
\end{aligned}
$$

The system (1) is then transformed to

$$
\begin{aligned}
\dot{\bar{x}} & =A_{c} \bar{x}+\phi(y) a+E(\omega)+d \xi_{1}, \\
y & =C \bar{x},
\end{aligned}
$$

where $d=\left(A_{c}+\lambda_{1} I\right) \bar{d}_{1}$. We then introduce another state transformation to extract the internal dynamics of (4) with $x \in \mathbb{R}^{n-1}$ given by $x=\bar{x}_{2: n}-\frac{d_{2: n}}{d_{1}} y$, where the notation $(\cdot)_{2: n}$ refers to the 2 nd row to the $n$th row of the corresponding vector. With the coordinates $(x, y),(4)$ is rewritten as

$$
\begin{aligned}
& \dot{x}=D x+\Xi y+\Omega_{1}(y, d) a+\tilde{E}(\omega, d), \\
& \dot{y}=x_{1}+\frac{d_{2}}{d_{1}} y+\phi_{1}(y) a+E_{1}(\omega)+b_{\rho} \xi_{1},
\end{aligned}
$$

where $D$ is the companion matrix of $d$ given by

$$
D=\left[\begin{array}{ccccc}
-d_{2} / d_{1} & 1 & 0 & \ldots & 0 \\
-d_{3} / d_{1} & 0 & 1 & \ldots & 0 \\
\vdots & \vdots & \vdots & \vdots & \vdots \\
-d_{n-1} / d_{1} & 0 & 0 & \ldots & 1 \\
-d_{n} / d_{1} & 0 & 0 & \ldots & 0
\end{array}\right]
$$

and $\Xi, \Omega_{1}(y, d), \tilde{E}(\omega, d)$ consist of system parameters. Notice that $\Omega_{1}(y, d)$ is dependent on $\phi(y)$, thus it is easy to check that $\Omega_{1}(0, d)=0$.

Internal model. The invariant manifold theory and internal model principle play a crucial role in establishing the solvability of the nonlinear output regulation problem.

Assumption 3. There exists an invariant manifold $\pi(\omega) \in \mathbb{R}^{n-1}$ satisfying

$$
\frac{\partial \pi(\omega)}{\partial \omega} s(\omega)=D \pi(\omega)+\psi(q(\omega), \omega, \mu),
$$

where $\psi(q(\omega), \omega, \mu)=\Xi q(\omega)+\Omega_{1}(q(\omega), d) a+$ $\tilde{E}(\omega, d), \mu=\left[a^{T}, b^{T}\right]^{T}$. Based on (7) and the second equation of (5), we have

$$
\frac{\partial q(\omega)}{\partial \omega} s(\omega)=\pi_{1}+\psi_{y}(q(\omega), \omega, \mu)+b_{\rho} \alpha(\omega),
$$

where $\pi_{1}$ is the first state of the invariant manifold $\pi, \quad \psi_{y}(q(\omega), \omega, \mu)=\frac{d_{2}}{d_{1}} q(\omega)+\phi_{1}(q(\omega)) a+E_{1}(\omega)$, $\alpha$ is the feedforward control input for disturbance suppression, and it refers to

$$
\alpha(\omega)=b_{\rho}^{-1}\left(\frac{\partial q(\omega)}{\partial \omega} s(\omega)-\pi_{1}-\psi_{y}(q(\omega), \omega, \mu)\right) .
$$

Let $\tilde{z}=x-\pi(\omega)$, then from equations (5), (7) and (8), the system can be represented as

$$
\begin{aligned}
\dot{\tilde{z}}= & D \tilde{z}+\Xi e+\Omega(y, \omega, d) a, \\
\dot{e}= & \tilde{z}_{1}+\frac{d_{2}}{d_{1}} e+\left(\phi_{1}(y)-\phi_{1}(q(\omega))\right) a, \\
& +b_{\rho}\left(\xi_{1}-\alpha(\omega)\right),
\end{aligned}
$$

where

$\Omega(y, \omega, d)=\phi_{2: n}(y)-\phi_{2: n}(q(\omega))-\frac{d_{2: n}}{d_{1}}\left(\phi_{1}(y)-\phi_{1}(q(\omega))\right)$.

The output regulation problem of system (1) degenerates to the stabilization problem of system (10).

Assumption 4. There exists an immersion of the exosystem

$$
\begin{aligned}
\dot{\eta} & =F \eta+G \gamma(H \eta), \\
\alpha(\omega) & =J \eta,
\end{aligned}
$$


where $\eta \in \mathbb{R}^{n_{r}}, J=[1,0, \ldots, 0]$, the pair $(F, J)$ is detectable, $\gamma(\cdot)$ is locally Lipschitz and is nondecreasing, that is, for all $\varrho_{1}, \varrho_{2} \in \mathbb{R}^{n_{r}}$, it satisfies $\left(\varrho_{1}-\varrho_{2}\right)^{T}\left[\gamma\left(\varrho_{1}\right)-\gamma\left(\varrho_{2}\right)\right] \geqslant 0$.

Remark 1. Assumption 4 is inspired by [6], this immersion system and the circle criterion are exploited for the design of internal model and estimated feedforward term $\alpha$. Besides, the system (11) is a virtual internal model, which is not related to the original system. Hence, the dimension of the internal model can be different from that of the original system. In this letter, we assume $\eta \in \mathbb{R}^{n_{r}}$.

Remark 2. In terms of Assumption 4, the internal model is a dynamic compensator independent of the uncertain parameters $\omega$ and can asymptotically reproduce the feedforward term $\alpha$. If the exosystem is linear, there always exists an immersion, and therefore the ideal feedforward control $\alpha$ can be reproduced under certain conditions. However, when the exosystem is nonlinear, there is no guarantee that there is an immersion in general, and further research on this topic is challenging. This is the reason why Assumption 4 is needed for the proposed algorithm. Furthermore, the conditions can be directly checked for the existence of the internal model with nonlinear exosystems.

Based on the parameterization (11), we design an internal model as $\dot{\hat{\eta}}=(F-K J)\left(\hat{\eta}-b_{\rho}^{-1} K e\right)+$ $G \gamma\left(H\left(\hat{\eta}-b_{\rho}^{-1} K e\right)\right)+K \xi_{1}$, where $K \in \mathbb{R}^{n_{r}}$ is chosen such that $F-K J$ is Hurwitz. If we define the auxiliary error $\tilde{\eta}=\eta-\hat{\eta}+b_{\rho}^{-1} K e$, then $\dot{\tilde{\eta}}=(F-K J) \tilde{\eta}+G \varphi(t, \psi)+\Upsilon, \Psi=H \tilde{\eta}$. According to the circle criterion, there exist a positive definite matrix $P_{\eta}$ and a semi-positive definite matrix $Q$ satisfying $(F-K J)^{T} P_{\eta}+P_{\eta}(F-K J)=$ $-Q, P_{\eta} G+H^{T}=0$ and $\eta^{T} Q \eta \geqslant \gamma_{0}\left|\eta_{1}\right|^{2}, \gamma_{0}>$ $0, \operatorname{span}\left(P_{\eta} K\right) \subseteq \operatorname{span}(Q)$.

Control design. Define $z_{1}=e, z_{i}=\xi_{i-1}-$ $\beta_{i-1}$, for $i=2, \ldots, \rho$ and $z_{\rho+1}=u-\beta_{\rho}$, where $\beta_{i}$ for $i=1, \ldots, \rho$ are stabilizing functions to be designed. Since the sign of the highfrequency gain $b_{\rho}$ is unknown, which is the different from [7]. A Nussbaum gain $N(\kappa)$ should be employed in the stabilizing function $\beta_{1}$. Let $\beta_{1}=N(\kappa) \bar{\beta}_{1}$ and $\dot{\kappa}=e \bar{\beta}_{1}, \kappa(0)=0$, where the Nussbaum gain $N$ is a function (e.g., $N(\kappa)=$ $\left.\kappa^{2} \cos \kappa\right)$ that satisfies the two-sided Nussbaum properties: $\quad \lim _{\kappa \rightarrow \pm \infty} \sup \frac{1}{\kappa} \int_{0}^{\kappa} N(s) d s=+\infty$ and $\lim _{\kappa \rightarrow \pm \infty} \inf \frac{1}{\kappa} \int_{0}^{\kappa} N(s) d s=-\infty$, where $\kappa \rightarrow \pm \infty$ denotes $\kappa \rightarrow+\infty$ and $\kappa \rightarrow-\infty$ respectively. Based on the adaptive backstepping, the design of $\beta_{i}$,
$2 \leqslant i \leqslant \rho$ is given by

$$
\begin{aligned}
\beta_{i}= & \lambda_{i-1} \xi_{i-1}-z_{i-1}-c_{i} z_{i}-k_{i}\left(\frac{\partial \beta_{i-1}}{\partial e}\right)^{2} z_{i} \\
& +\frac{\partial \beta_{i-1}}{\partial \hat{b}_{\rho}} \tau_{i}+\chi_{i}+\frac{\partial \beta_{i-1}}{\partial \hat{\eta}} \dot{\hat{\eta}}+\frac{\partial \beta_{i-1}}{\partial \kappa} \dot{\kappa} \\
& +\sum_{j=1}^{i-2} \frac{\partial \beta_{i-1}}{\partial \xi_{j}}\left(-\lambda_{j} \xi_{j}+\xi_{j+1}\right) \\
& +\frac{\partial \beta_{i-1}}{\partial e}\left(\hat{b}_{\rho}\left(\xi_{1}-\hat{\eta}_{1}\right)+K_{1} e\right) .
\end{aligned}
$$

The detailed proof of this result is provided in the Appendixes A-D. We then design the control input by setting $z_{\rho+1}=0, u=\beta_{\rho}$.

Conclusion. In this letter, the adaptive output regulation problem for nonlinear systems in output feedback form proposed in [7] has been considered without the knowledge of the high-frequency gain. Filtered transformation has been used to deal with high relative degrees. The proposed internal model, which is under some conditions, is designed by the circle criterion. The success of the introduction of the Nussbaum gain together with the internal model makes it possible to deduce the adaptive laws, and by using adaptive backstepping, the control input has been obtained.

Supporting information Appendixes A-D. The supporting information is available online at info. scichina.com and link.springer.com. The supporting materials are published as submitted, without typesetting or editing. The responsibility for scientific accuracy and content remains entirely with the authors.

\section{References}

1 Chen Z Y, Huang J. Stabilization and regulation of nonlinear systems. Springer, 2015

2 Riccardo M, Patrizio T. Global adaptive outputfeedback control of nonlinear systems, Part I: Linear parameterization. IEEE Transactions on Automatic Control, 1993, 38: 17-32

3 Chen Z Y, Huang J. Attitude tracking of rigid spacecraft subject to disturbances of unknown frequencies. International Journal of Robust and Nonlinear Control, 2014, 24: 2231-2242

4 Ding Z T. Adaptive consensus output regulation of a class of nonlinear systems with unknown highfrequency gain. Automatica, 2015, 51: 348-355

$5 \mathrm{Yu}$ W W, Wang H, Hong H F, et al. Distributed cooperative anti-disturbance control of multi-agent systems: an overview. Sci China Inf Sci, 2017, 60(11): 110202

6 Guo M C, Xu D B, Liu L. A result on output regulation of lower triangular systems with unknown highfrequency gain sign. International Journal of Robust and Nonlinear Control, 2017, 27: 4903-4918

7 Xi Z R, Ding Z T. Global adaptive output regulation of a class of nonliner systems with nonlinear exosystems. Automatica, 2007, 43: 143-149 